\title{
Juvenile-onset obesity differentially affects hepatic glucocorticoid, growth hormone (GH), insulin-like growth factor (IGF)-1 and insulin sensitivity in 1-year-old sheep
}

\author{
Melanie A. Hyatt ${ }^{1}$, Natasha Davidson ${ }^{1}$, Sylvain Sebert ${ }^{1}$, Laureen Chan ${ }^{1}$, Terence Stephenson ${ }^{1}$, \\ Michael Symonds ${ }^{1}$, David Gardner ${ }^{2}$ and Helen Budge ${ }^{1}$ \\ ${ }^{1}$ Centre for Reproduction and Early Life, University of Nottingham, Nottingham, UK and ${ }^{2}$ School of Veterinary Medicine \\ and Science, University of Nottingham, Nottingham, UK
}

In human subjects chronic energy excess combined with sedentary behaviour causes obesity, which is a major determinant of insulin resistance and hyperinsulinaemia; disorders strongly associated with the development of the metabolic syndrome ${ }^{(1)}$. There is increasing evidence that glucocorticoids together with the GH-IGF axis play an important role in obesity and insulin resistance leading to type 2 diabetes. A sheep model of juvenile obesity has previously been developed that incorporates both increased energy intake with reduced physical activity ${ }^{(2)}$. The present study aimed to determine the extent to which hepatic insulin and glucocorticoid sensitivity and expression of the GH-IGF axis are reset by juvenile obesity in the sheep.

Twenty-three pregnant sheep were randomly assigned to either a control diet (approximately $7 \mathrm{MJ} / \mathrm{d}$ ) or a nutrient-restricted diet (approximately $3.5 \mathrm{MJ} / \mathrm{d}$ ) from $30 \mathrm{~d}$ to $80 \mathrm{~d}$ gestation (term $147 \mathrm{~d}$ ) and were fed to requirements at all other times. Following weaning at 10 weeks postnatal age, control offspring were randomly assigned to either a lean $(\mathrm{L} ; n 6)$ or obeseogenic $(\mathrm{O} ; n$ 7) environment. NR offspring were also exposed to an obesogenic environment (i.e. NR-O). Group $\mathrm{O}$ and NR-O offspring were fed energy-dense food in a restricted physical activity environment to promote fat deposition and juvenile obesity, whilst group L remained on pasture and had unrestricted physical activity. At 1 year of age fasted blood samples were taken for measurement of plasma glucose and cortisol concentrations. Adult offspring were humanely killed by electrocortical stunning and exsanguination at 1 year of age to enable liver tissue sampling. Animal procedures were approved by the local Animal Ethics Committee and were performed in accordance with UK legislation. Plasma glucose and cortisol concentrations were measured by spectrophotometric methods and RIA respectively. mRNA abundance of target genes was determined using fully-validated real-time RT-PCR techniques. Results, in arbitrary units (au), were calculated using the $2^{-\Delta \mathrm{CT}}$ method and are expressed relative to group $\mathrm{L}^{(3)}$. Significant differences $(P<0.05)$ in relation to the prenatal diet and post-weaning obesity were determined using Student's independent $t$ tests.

Juvenile-onset obesity and prenatal diet had no effect on relative liver weight, plasma glucose and cortisol concentrations or insulin receptor mRNA abundance. However, obesity significantly reduced hepatic gene expression of GH receptor, IGF-1 receptor, and PPAR $\gamma$ coactivator-1 $\alpha$. Whilst, glucocorticoid receptor mRNA was significantly increased in the livers of obese sheep. Prenatal diet had no effect on hepatic gene expression (Table).

Table. Effects of maternal nutrient restriction and juvenile onset obesity

\begin{tabular}{|c|c|c|c|c|c|c|c|c|}
\hline & \multicolumn{2}{|c|}{$\mathrm{L}$} & \multicolumn{2}{|c|}{$\mathrm{O}$} & \multicolumn{2}{|c|}{ NR-O } & \multicolumn{2}{|c|}{$P$} \\
\hline & Mean & $\mathrm{SE}$ & Mean & $\mathrm{SE}$ & Mean & $\mathrm{SE}$ & $\mathrm{L} v . \mathrm{O}$ & $\mathrm{O} v . \mathrm{NR}-\mathrm{O}$ \\
\hline Liver $(\mathrm{g} / \mathrm{kg})$ & 10.4 & 0.3 & 9.9 & 0.6 & 10.2 & 0.5 & NS & NS \\
\hline Glucose $(\mathrm{mmol} / \mathrm{l})$ & 4.5 & 0.2 & 5.9 & 0.7 & 5.4 & 0.7 & NS & NS \\
\hline Cortisol (nmol/l) & 58.6 & 8.9 & 76.6 & 17.1 & 89.9 & 14.8 & & \\
\hline Hepatic mRNA abundan & & & & & & & & \\
\hline Insulin receptor & 1.0 & 0.4 & 0.4 & 0.1 & 0.2 & 0.02 & $P<0.05$ & NS \\
\hline GH receptor & 1.0 & 0.2 & 0.4 & 0.1 & 0.4 & 0.09 & $P<0.05$ & NS \\
\hline IGF-I receptor & 1.0 & 0.2 & 0.3 & 0.05 & 0.6 & 0.2 & $P<0.05$ & NS \\
\hline PPAR $\gamma$ coactivator- $1 \alpha$ & 1.0 & 0.1 & 0.3 & 0.03 & 0.4 & 0.1 & $P<0.05$ & NS \\
\hline Glucocorticoid receptor & 1.0 & 0.2 & 3.3 & 1.1 & 2.9 & 0.6 & $P<0.05$ & NS \\
\hline
\end{tabular}

Obesity resulted in decreased GH receptor and IGF-1 receptor abundance, signifying a state of hepatic GH resistance and insulin insensitivity, which are suggestive of impaired metabolic function, possibly in response to increased tissue glucocorticoid sensitivity. However, this outcome is unlikely to have a gluconeogenic effect as obese animals remained normoglycaemic. Further to alterations in the hepatic glucocorticoid-GH-IGF axis, obesity induced a reduction in PPAR $\gamma$ coactivator-1 $\alpha$. This effect may indicate a decrease in hepatic fatty acid oxidation and perturbed lipid metabolism, which may be indicative of precocious non-alcoholic fatty liver disease.

1. Cheal KL, Abbasi F, Lamendola C, McLaughlin T, Reaven GM \& Ford ES (2004) Diabetes 53, 1195-1200.

2. Williams PJ, Kurlak LO, Perkins AC, Budge H, Stephenson T, Keisler D, Symonds ME \& Gardner DS (2007) Kidney Int 72, $279-289$.

3. Livak KJ \& Schmittgen TD (2001) Methods 25, 402-408. 